\title{
Correction to: Clinical Practice Guidelines for Childbearing Female Candidates for Bariatric Surgery, Pregnancy, and Post-Partum Management after Bariatric Surgery
}

Cécile Ciangura $^{1}$ - Muriel Coupaye ${ }^{2,3} \cdot$ Philippe Deruelle $^{4,5} \cdot$ Géraldine Gascoin $^{6,7}$ - Daniela Calabrese ${ }^{8}$. Emmanuel Cosson ${ }^{9,10}$. Guillaume Ducarme ${ }^{11}$ - Bénédicte Gaborit ${ }^{3,12} \cdot$ Bénédicte Lelièvre $^{13} \cdot$ Laurent Mandelbrot $^{14}$. Niccolo Petrucciani ${ }^{15}$ - Didier Quilliot ${ }^{16,17}$. Patrick Ritz ${ }^{18} \cdot$ Geoffroy Robin $^{5,19}$ • Agnès Sallé ${ }^{20}$. Jean Gugenheim ${ }^{21,22}$. Jacky Nizard ${ }^{23} \cdot$ BARIA-MAT Group

Published online: 5 June 2020

(C) Springer Science+Business Media, LLC, part of Springer Nature 2020

Correction to: Obesity Surgery (2019) 29:3722-3734 https://doi.org/10.1007/s11695-019-04093-y

In the original article, due to an XML tagging error the name of Véronique Taillard was omitted from the list of members of the French Study Group for Bariatric Surgery and Maternity (the BARIA-MAT Group). The correct list is as follows:

French Study Group for Bariatric Surgery and Maternity including all listed authors and Virginie Castera, $\mathrm{MD}^{1}$, Régis Coutant, $\mathrm{MD}, \mathrm{PhD}^{2}$, Thierry Dupré, $\mathrm{MD}^{3}$, Hubert Johanet,

The online version of the original article can be found at https://doi.org/ 10.1007/s11695-019-04093-y

\author{
Cécile Ciangura \\ cecile.ciangura@aphp.fr \\ Muriel Coupaye \\ muriel.coupaye@aphp.fr \\ Philippe Deruelle \\ pderuelle@unistra.fr \\ Géraldine Gascoin \\ GeGascoin@chu-angers.fr \\ Daniela Calabrese \\ daniela.calabrese@aphp.fr \\ Emmanuel Cosson \\ emmanuel.cosson@aphp.fr \\ Guillaume Ducarme \\ g.ducarme@gmail.com \\ Bénédicte Gaborit \\ Benedicte.GABORIT@ap-hm.fr \\ Bénédicte Lelièvre \\ BeLelievre@chu-angers.fr
}

$\mathrm{MD}^{4}$, Marie Pigeyre, $\mathrm{MD}, \mathrm{PhD}^{5}$, Brigitte Rochereau, $\mathrm{MD}^{6}$, Véronique Taillard, $\mathrm{MD}^{7}$, Claudine Canale ${ }^{8}$, Anne-Sophie Joly ${ }^{8}$.

${ }^{1}$ Department of Endocrinology, St Joseph Hospital, Marseille, France

\author{
Laurent Mandelbrot \\ laurent.mandelbrot@aphp.fr \\ Niccolo Petrucciani \\ nicpetrucciani@hotmail.it \\ Didier Quilliot \\ quilliot.d@orange.fr \\ Patrick Ritz \\ patrick.ritz@wanadoo.fr \\ Geoffroy Robin \\ Geoffroy.ROBIN@CHRU-LILLE.fr \\ Agnès Sallé \\ AgSalle@chu-angers.fr \\ Jean Gugenheim \\ gugenheim.j@chu-nice.fr \\ Jacky Nizard \\ jacky.nizard@aphp.fr
}

Extended author information available on the last page of the article 
${ }^{2}$ Department of Pediatrics, Angers University Hospital, Angers, France

${ }^{3}$ Assistance Publique-Hôpitaux de Paris, Bichat Hospital,

Department of Biochemistry, Paris, France; Société

Française des Vitamines et Biofacteurs

${ }^{4}$ Clinique Turin, Paris, France
${ }^{5}$ Department of Nutrition, CHRU Lille, University Lille, Lille, France

${ }^{6}$ Antony, France

${ }^{7}$ Department of Nutrition, CHU Nimes, Nimes, France

${ }^{8}$ Collectif National des Associations d'Obèses (CNAO)

\section{Affiliations}

\section{Cécile Ciangura ${ }^{1} \cdot$ Muriel Coupaye $^{2,3} \cdot$ Philippe Deruelle $^{4,5} \cdot$ Géraldine Gascoin $^{6,7}$ - Daniela Calabrese ${ }^{8}$. Emmanuel Cosson ${ }^{9,10}$. Guillaume Ducarme ${ }^{11}$ - Bénédicte Gaborit ${ }^{3,12}$ - Bénédicte Lelièvre ${ }^{13} \cdot$ Laurent Mandelbrot $^{14}$. Niccolo Petrucciani ${ }^{15}$. Didier Quilliot ${ }^{16,17}$. Patrick Ritz ${ }^{18}$. Geoffroy Robin ${ }^{5,19}$ - Agnès Sallé ${ }^{20} \cdot$ Jean Gugenheim ${ }^{21,22}$. Jacky Nizard ${ }^{23}$ • BARIA-MAT Group}

1 Assistance Publique-Hôpitaux de Paris, Pitié-Salpêtrière Hospital, Departments of Nutrition and Diabetology, Institute of Cardiometabolism and Nutrition (ICAN), Sorbonne University, 4783 Boulevard de l'Hôpital, 75013 Paris, France

2 Assistance Publique-Hôpitaux de Paris, Louis Mourier Hospital, Department of Explorations Fonctionnelles, Colombes, University Paris Diderot, Paris, France

3 Association Française d'Etude et de Recherche sur l'Obésité (AFERO), Paris, France

4 Department of Obstetrics and Gynecology, Strasbourg University Hospital, Strasbourg, France

5 Collège National des Gynécologues et Obstétriciens Français, (CNGOF), Paris, France

6 Department of Neonatal Medicine, Angers University Hospital, Angers, France

7 Société Française de Néonatologie et Société Française de Pédiatrie, Paris, France

8 Assistance Publique-Hôpitaux de Paris, Louis Mourier Hospital, Department of Digestive Surgery, Colombes, University Paris Diderot, Paris, France

9 Assistance Publique-Hôpitaux de Paris, Jean Verdier Hospital, Department of Endocrinology-Diabetology-Nutrition, Bondy, 10 UMR U557 INSERM/U11125 INRA/CNAM, University Paris13, Bobigny, France

10 Société Francophone du Diabète (SFD), Paris, France

11 Department of Obstetrics and Gynecology, Departemental Hospital, La Roche-sur-Yon, France

12 Assistance Publique-Hôpitaux de Marseille, Department of Endocrinology, Metabolic Diseases and Nutrition, Marseille,
INSERM, INRA, C2VN, Aix Marseille University, Marseille, France

13 Laboratory of Pharmacology and Toxicology, Angers University Hospital, Angers, France

14 Assistance Publique-Hôpitaux de Paris, Louis Mourier Hospital, Department of Gynecology and Obstetrics, Colombes, University Paris Diderot, Paris, France

15 Assistance Publique-Hôpitaux de Paris, Henri Mondor Hospital, Division of Digestive Surgery and Liver Transplantation, UPEC University, Créteil, France

16 Department of Diabetology-Endocrinology-Nutrition, CHRU Nancy, INSERM 954, University of Lorraine, Nancy, France

17 Société Francophone Nutrition Clinique et Métabolisme (SFNCM), Paris, France

18 Department of Endocrinology, Metabolic Diseases and Nutrition, Toulouse University Hospital, UMR1027, Paul Sabatier University, Toulouse, France

19 Department of Medical Gynecology, and Sexology and Department of Assisted Reproductive Technologies and Fertility Preservation, Jeanne de Flandre Hospital, Lille, EA4308 "gametogenesis and gamete quality", Lille University, Lille, France

20 Department of Diabetology-Endocrinology-Nutrition, Angers University Hospital, Angers, France

21 Division of Digestive Surgery and Liver Transplantation, Nice University Hospital, University of Nice, Nice, France

22 Porte des Pierres, Dorées, France

23 Assistance Publique-Hôpitaux de Paris, Pitié-Salpêtrière Hospital, Department of Gynecology and Obstetrics, Sorbonne University, Paris, France 